\title{
On the reduction of pairs of bounded closed convex sets
}

\author{
by \\ J. Grzybowski (Poznań), D. Pallaschke (Karlsruhe) and \\ R. URBAŃSKi (Poznań)
}

\begin{abstract}
Let $X$ be a Hausdorff topological vector space. For nonempty bounded closed convex sets $A, B, C, D \subset X$ we denote by $A \dot{+} B$ the closure of the algebraic sum $A+B$, and call the pairs $(A, B)$ and $(C, D)$ equivalent if $A \dot{+} D=B \dot{+} C$. We prove two main theorems on reduction of equivalent pairs. The first theorem implies that, in a finite-dimensional space, a pair of nonempty compact convex sets with a piecewise smooth boundary and parallel tangent spaces at some boundary points is not minimal. The second theorem generalizes and unifies two main techniques of reduction of pairs of compact convex sets.
\end{abstract}

1. Introduction. Let $X$ be a locally convex vector space and denote by $\mathcal{B}(X)$ the set of all nonempty bounded closed convex subsets of $X$. L. Hörmander [7] and H. Rådström [15] investigated the space $\mathcal{B}^{2}(X) / \sim$, where $\mathcal{B}^{2}(X)=\mathcal{B}(X) \times \mathcal{B}(X)$ and the equivalence relation is defined by

where

$$
(A, B) \sim(C, D) \Leftrightarrow A \dot{+} D=B \dot{+} C,
$$

$$
A+B=\operatorname{cl}(\{x=a+b \mid a \in A \text { and } b \in B\})
$$

is the Minkowski sum, which is the closure of the algebraic sum of $A$ and $B$. The quotient space $\mathcal{B}^{2}(X) / \sim$ is a linear space, called the Hörmander-Rådström lattice. The element of $\mathcal{B}^{2}(X) / \sim$ which contains the pair $(A, B)$ is denoted by $[A, B]$.

There exists an interesting application of the Hörmander-Rådström lattice in the quasidifferentiable calculus of V. F. Demyanov and A. M. Rubinov (see [1], [2] and [9]). For a large class of nonsmooth functions, at any point of the domain of the function one defines a set of generalized gradients which is an element of the Hörmander-Rådström lattice.

This application gave rise to the investigation of inclusion minimal representatives for elements of the Rådström-Hörmander lattice. Let us introduce

2000 Mathematics Subject Classification: 52A07, 26A51, 49J52.

Key words and phrases: convex analysis, separation law, pairs of bounded closed convex sets. 
the following order:

$$
(A, B) \leq(C, D) \Leftrightarrow A \subset C \text { and } B \subset D .
$$

Then $\left(A_{0}, B_{0}\right) \in[A, B]$ is an inclusion minimal representative of $[A, B]$ if it is a minimal element in $[A, B]$. We also call $\left(A_{0}, B_{0}\right)$ a minimal pair.

A key to the investigation of inclusion minimal representatives is the order cancellation law, first proved by H. Rådström [15] for nonempty compact convex sets, and by R. Urbański [18] for all nonempty bounded closed convex sets. Because of its frequent use in this paper, we recall it here:

TheOREM 1.1 (Order cancellation law). Let $X$ be a topological vector space. Then for $A, B, C \in \mathcal{B}(X)$ we have the implication

$$
A \dot{+} B \subset C \dot{+} B \Rightarrow A \subset C .
$$

A set $A \in \mathcal{B}(X)$ is called a summand of $B \in \mathcal{B}(X)$ if there exists $C \in \mathcal{B}(X)$ such that $A \dot{+} C=B$. Obviously the following characterization of inclusion minimal representatives follows from the order cancellation law:

The pair $\left(A_{0}, B_{0}\right) \in[A, B]$ is an inclusion minimal representative if and only if there exists no proper bounded closed convex subset $K \subset A_{0} \dot{+} B_{0}$ such that $A_{0}$ and $B_{0}$ are summands of $K$.

Minimal pairs have many interesting properties which have been studied in a series of papers (see [3]-[6], [10]-[13] and [17]). For instance in the 2 -dimensional case, equivalent minimal pairs of compact convex sets are uniquely determined up to translations (see [4], [17]), which is no longer true in the 3-dimensional case. A continuous family of equivalent minimal pairs of compact convex sets which are not related by a translation is given in [11]. J. Grzybowski and R. Urbański [6] showed, under the assumption of the continuum hypothesis, that if there exist two equivalent minimal pairs of compact convex sets which are not related by a translation, then there exists a continuous family of equivalent minimal pairs which are also not related by translations.

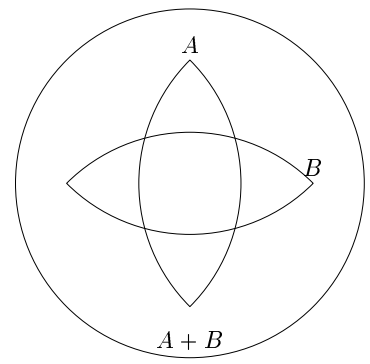

Fig. 1.1 
An interesting example is the pair $(A, B)$ of two orthogonal lenses in $\mathbb{R}^{2}$. It is known (see [13]) that their Minkowski sum is the unit disk and that there exists no proper convex subset $K$ of the unit disk such that both $A$ and $B$ are summands of $K$.

Hence the pair $(A, B)$ is minimal. It follows from a more sophisticated argument (see [13, Chapter 4]) that the pair $(A, C)$, where $C$ arises by turning $B$ through $\frac{\pi}{4}$, is not minimal.

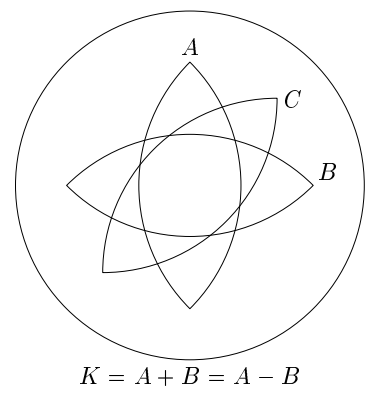

Fig. 1.2

This is proved by the reduction method for pairs of compact convex sets (see [13, Theorem 4.7.3]), according to which all parts of these two sets which can be translated onto each other can be cut off (see Figure 1.3).

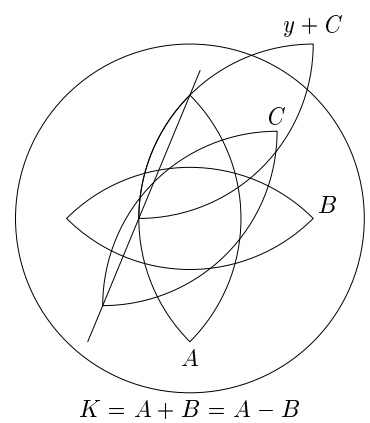

Fig. 1.3

The main topic of this paper is the investigation of pairs of convex sets with smooth touching boundaries.

2. Notations and properties of convex sets. For a topological vector space $X$ let us denote by $\mathcal{K}(X) \subset \mathcal{B}(X)$ the set of all nonempty compact convex subsets of $X$. For $A \in \mathcal{B}(X)$ and $\lambda \in \mathbb{R}$ we put $\lambda A=\{x=\lambda a \mid$ $a \in A\}$. Moreover for $A, B \in \mathcal{B}(X)$ we define $A \vee B=\operatorname{cl} \operatorname{conv}(A \cup B)$, where "cl conv" denotes the closed convex hull. It is easy to see that $A \vee B$ is the 
supremum of $A, B \in \mathcal{B}(X)$ with respect to the order given by inclusion. Moreover, for a subset $A \subset X$, we denote by $\partial A$ the boundary of $A$.

Observe that for compact convex sets, the Minkowski sum coincides with the algebraic sum, i.e., for $A, B \in \mathcal{K}(X)$ we have $A \dot{+} B=A+B$.

We will use the abbreviation $A \dot{+} B \vee C$ for $A \dot{+}(B \vee C)$, and $C+d$ for $C+\{d\}$, for any $A, B, C \in \mathcal{B}(X)$ and $d \in X$. For $a, b \in X$ the interval with end points $a$ and $b$ will be denoted by $[a, b]=\{a\} \vee\{b\}$.

A convex subset $B$ of a convex set $A \subset X$ is called an extreme subset if for any $x, y \in A$ the condition $t x+(1-t) y \in B$ for some $t \in(0,1)$ implies that $x, y \in B$. An extreme subset which consists of a single point is called an extreme point, and $\mathcal{E}(A)$ denotes the set of extreme points of $A$.

A convex set which is the convex hull of finitely many points is called a polytope. The set of all polytopes of a vector space $X$ is denoted by $\mathcal{P}(X)$. An extreme subset of a polytope is called a face.

If $X$ is a topological vector space and $X^{*}$ its dual, then for $A \in \mathcal{K}(X)$ and $f \in X^{*}$ we denote by

$$
H_{f}(A)=\left\{z \in A \mid f(z)=\max _{y \in A} f(y)\right\}
$$

the (maximal) face of $A$ with respect to $f$.

We will now state some further fundamental properties of convex sets. We begin with the Pinsker formula (see [14], [13]), which states the distributivity between the supremum operation and the Minkowski sum.

Theorem 2.1 (Pinsker formula). Let $X$ be a topological vector space. Then for all $A, B, C \in \mathcal{B}(X)$,

$$
(A \dot{+} C) \vee(B \dot{+} C)=C \dot{+}(A \vee B) \text {. }
$$

From the order cancellation law and the Pinsker formula it follows that $(\mathcal{B}(X), \dot{+}, \leq)$ is an ordered commutative semigroup with respect to the Minkowski sum and the order induced by inclusion, which satisfies the order cancellation law. An abstract characterization of such semigroups is given in $[8]$ and $[16]$.

Let us now formulate the separation law. Let $A, B, S \in \mathcal{B}(X)$. The set $S$ is said to separate $A$ and $B$ if $[a, b] \cap S \neq \emptyset$ for all $a \in A$ and $b \in B$. This is a natural generalization of the separation of two disjoint sets by a hyperplane. Note that $A$ and $B$ may intersect. If $S$ separates $A$ and $B$, then $A \cap B \subset S$. The definition is illustrated in Figure 2.1.

TheOREM 2.2. Let $X$ be a topological vector space and $A, B \in \mathcal{B}(X)$. Then the following statements are equivalent:

(i) $A \cup B$ is convex.

(ii) $A \cap B$ separates $A$ and $B$.

(iii) $A \dot{+} B=A \vee B \dot{+} A \cap B$ and $A \cap B \neq \emptyset$. 


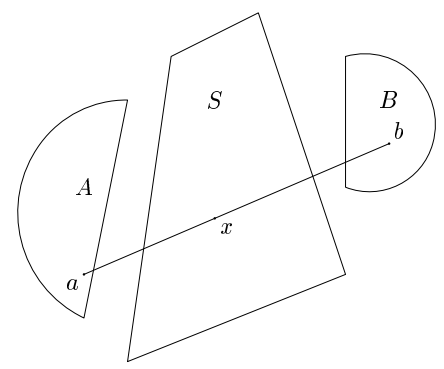

Fig. 2.1

Theorem 2.2 plays a central role in further investigations because it is an algebraic characterization of the geometric situation when the union of two bounded closed convex subsets is again convex, or equivalently, when their intersection separates both sets.

Theorem 2.3 (Separation law). Let $A, B$ be nonempty subsets of a topological vector space $X$ and assume that $A \vee B \in \mathcal{B}(X)$. Moreover, let $S \in \mathcal{B}(X)$. Then $S$ separates $A$ and $B$ if and only if

$$
A+B \subset A \vee B+S \text {. }
$$

It is proved in [12] (see also [13]) that the separation law is equivalent to the order cancellation law.

We now state another two useful properties. The first is the formula for maximal faces of the Minkowski sum (see [20], [13]):

Proposition 2.4. Let $X$ be a topological vector space, $f \in X^{*}$ and $A, B \in \mathcal{K}(X)$. Then

$$
H_{f}(A+B)=H_{f}(A)+H_{f}(B) .
$$

The other is the additivity of convex hull (see [13]):

Proposition 2.5. Let $X$ be a vector space and $A, B \subset X$. Then

$$
\operatorname{conv} A+\operatorname{conv} B=\operatorname{conv}(A+B) \text {. }
$$

3. Pairs with locally smooth boundaries. First we discuss an extension property for equivalent pairs of bounded closed convex sets. Given two equivalent pairs $\left(A_{1}, B_{1}\right)$ and $\left(A_{1}^{\prime}, B_{1}^{\prime}\right)$ of nonempty bounded closed convex sets. Then any two bounded closed convex sets $A_{2}$ and $B_{2}$ for which $\left(A_{1} \cup A_{2}, B_{1} \cup B_{2}\right)$ and $\left(A_{1}^{\prime} \cup A_{2}, B_{1}^{\prime} \cup B_{2}\right)$ are also pairs of bounded closed convex sets must satisfy condition (ii) of Theorem 2.2. If the separating intersections are equal, as in Figure 3.1, then the extended pairs $\left(A_{1} \cup A_{2}, B_{1} \cup B_{2}\right)$ and $\left(A_{1}^{\prime} \cup A_{2}, B_{1}^{\prime} \cup B_{2}\right)$ are also equivalent.

Proposition 3.1. Let $X$ be a topological vector space and let $A, A_{1}, A_{2}$, $A^{\prime}, A_{1}^{\prime}, B, B_{1}, B_{2}, B^{\prime}, B_{1}^{\prime} \in \mathcal{B}(X)$ be such that $A=A_{2} \cup A_{1}, A^{\prime}=A_{2} \cup A_{1}^{\prime}$, 

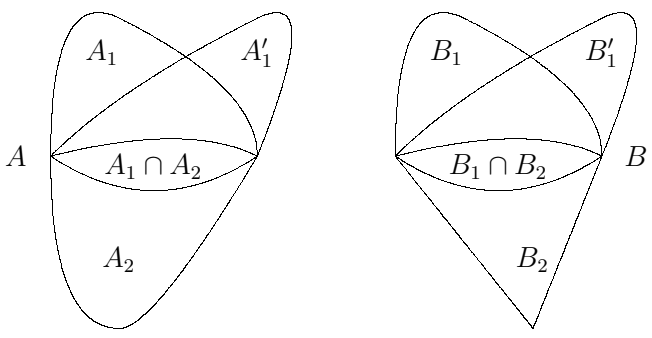

Fig. 3.1

$B=B_{2} \cup B_{1}$ and $B^{\prime}=B_{2} \cup B_{1}^{\prime}$. Moreover, assume that $A_{2} \cap A_{1}=A_{2} \cap A_{1}^{\prime}$ and $B_{2} \cap B_{1}=B_{2} \cap B_{1}^{\prime}$. If $\left(A_{1}, B_{1}\right) \sim\left(A_{1}^{\prime}, B_{1}^{\prime}\right)$, then $(A, B) \sim\left(A^{\prime}, B^{\prime}\right)$.

Proof. By Theorem 2.2 we have

$$
\begin{aligned}
A \dot{+} B^{\prime} \dot{+} A_{2} \cap A_{1} \dot{+} B \dot{+} B_{2} \cap B_{1} & =A_{2} \cup A_{1} \dot{+} A_{2} \cap A_{1} \dot{+} B_{2} \cup B_{1}^{\prime} \dot{+} B_{2} \cap B_{1}^{\prime} \\
& =A_{2} \dot{+} A_{1} \dot{+} B_{2} \dot{+} B_{1}^{\prime}=A_{2} \dot{+} A_{1}^{\prime} \dot{+} B_{2} \dot{+} B_{1} \\
& =A_{2} \cup A_{1}^{\prime} \dot{+} A_{2} \cap A_{1}^{\prime} \dot{+} B_{2} \cup B_{1} \dot{+} B_{2} \cap B_{1} \\
& =A^{\prime} \dot{+} B \dot{+} A_{2} \cap A_{1} \dot{+} B_{2} \cap B_{1} .
\end{aligned}
$$

Applying the cancellation law we obtain $A+B^{\prime}=A^{\prime} \dot{+} B$.

The situation of the next proposition is illustrated in Figure 3.2.

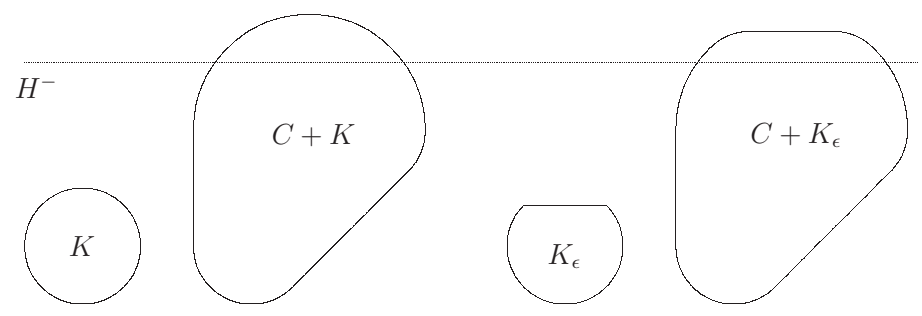

Fig. 3.2

Proposition 3.2. Let $C \in \mathcal{K}\left(\mathbb{R}^{n}\right)$ and $K=\mathbb{B}(0, r) \subset \mathbb{R}^{n}$ be the closed Euclidean ball with center 0 and radius $r>0$. Moreover, let $f \in\left(\mathbb{R}^{n}\right)^{*}$ with $\|f\|=1$ and $\max f(C+K)>0$. Put $H^{-}=f^{-1}((-\infty, 0])$. Then there exists an $\varepsilon>0$ such that $(C+K) \cap H^{-} \subset C+K_{\varepsilon}$, where $K_{\varepsilon}=\{x \in K \mid f(x) \leq$ $r-\varepsilon\}$.

Proof. First we will prove that there exists an $\varepsilon>0$ with $\partial(C+K) \cap$ $H^{-} \subset C+K_{\varepsilon}$.

Suppose that this is not true. Then for every $k \in \mathbb{N}$ there exists a point $x_{k} \in \partial(C+K) \cap H^{-}$which does not belong to $C+K_{1 / k}$. Moreover, there exists $f_{k} \in\left(\mathbb{R}^{n}\right)^{*}$ such that $x_{k} \in H_{f_{k}}(C+K)$. By Proposition 2.4 we have 
$H_{f_{k}}(C+K)=H_{f_{k}}(C)+H_{f_{k}}(K)$, where $H_{f_{k}}(K)$ is a singleton $\left\{y_{k}\right\}$, because the unit ball is strictly convex. Since $y_{k} \notin K_{1 / k}$ it follows that $y_{k}$ tends to $y$ with $\{y\}=H_{f}(K)$. From the strict convexity of the unit ball it also follows that $\bar{f}_{k}=\left\langle y_{k} / r, \cdot\right\rangle$ tends to $\bar{f}=\langle y / r, \cdot\rangle$.

Since $\partial(C+K) \cap H^{-}$is compact, there exists a sequence $\left(k_{m}\right)$ such that $x_{k_{m}}$ tends to some $x \in \partial(C+K) \cap H^{-}$. Note that $f_{k_{m}}=\max f_{k_{m}}(C+K)$ tends to both $f(x)$ and $\max f(C+K)$. Hence $f(x)>0$, which contradicts the fact that $x \in H^{-}$.

Now let $x \in(C+K) \cap H^{-}$and put $H^{x}=f^{-1}(f(x))$. Then $A=(C+K)$ $\cap H^{x}$ is a nonempty compact convex subset of the affine hyperplane $H^{x}$. Since $A$ is equal to the convex hull of $\partial A$, and $\partial A \subset \partial(C+K) \cap H^{-}$, it follows that $x \in \operatorname{conv}\left(\partial(C+K) \cap H^{-}\right)$. Therefore, $(C+K) \cap H^{-} \subset C+K_{\varepsilon}$.

Theorem 3.3. Let $A, A_{1}, B, B_{1}, C, D \in \mathcal{K}\left(\mathbb{R}^{n}\right), K=\mathbb{B}(0, r)$ and let $f \in\left(\mathbb{R}^{n}\right)^{*}$ with $\|f\|=1, \max f(A)>0$ and $\max f(B)>0$. Put $H^{-}=$ $f^{-1}((-\infty, 0])$. Assume that $A_{1}=A \cap H^{-}, B_{1}=B \cap H^{-}, A=A_{1} \cup(C+K)$ and $B=B_{1} \cup(D+K)$. Then the pair $(A, B)$ is not minimal.

Proof. Applying Proposition 3.2 we obtain $(C+K) \cap H^{-} \subset C+K_{\varepsilon}$ and $(D+K) \cap H^{-} \subset D+K_{\varepsilon}$ for some $\varepsilon>0$, with $K_{\varepsilon}=\{x \in K \mid f(x) \leq r-\varepsilon\}$. Now put $A_{2}=C+K, A_{2}^{\prime}=C+K_{\varepsilon}, B_{2}=D+K$ and $B_{2}^{\prime}=D+K_{\varepsilon}$. Then $A_{1} \cap A_{2}=A_{1} \cap A_{2}^{\prime}, B_{1} \cap B_{2}=B_{1} \cap B_{2}^{\prime}$ and $\left(A_{2}, B_{2}\right) \sim(C, D) \sim\left(A_{2}^{\prime}, B_{2}^{\prime}\right)$. Applying Proposition 3.1 we obtain $(A, B) \sim\left(A^{\prime}, B^{\prime}\right)$. Since $A^{\prime} \subset A$ and

$\max f\left(A^{\prime}\right)=\max f(C)+\max f\left(K_{\varepsilon}\right)<\max f(C)+\max f(K)=f(A)$, it follows that $A^{\prime} \neq A$ and so the pair $(A, B)$ is not minimal.

The geometric meaning of Theorem 3.3 is shown in Figure 3.3.

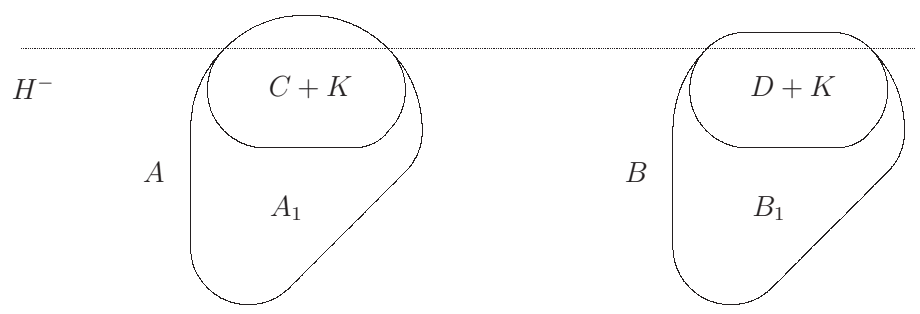

Fig. 3.3

Definition 3.4. A set $A \in \mathcal{K}\left(\mathbb{R}^{n}\right)$ is called locally $\varepsilon$-smooth at $x_{0} \in \partial A$ if there exists a neighborhood $U$ of $x_{0}$ such that for every $x \in A \cap U$ there exists $y \in \mathbb{R}^{n}$ with $x \in y+\varepsilon \mathbb{B}^{n} \subset A$, where $\mathbb{B}^{n}=\mathbb{B}(0,1) \subset \mathbb{R}^{n}$.

From Theorem 3.3 we deduce the first main result: 
Theorem 3.5. Let $A, B \in \mathcal{K}\left(\mathbb{R}^{n}\right)$ and let $A$ be locally $\varepsilon$-smooth at $x_{0} \in$ $\partial A$ and $B$ be locally $\varepsilon$-smooth at $y_{0} \in \partial B$. If there exists an $f \in\left(\mathbb{R}^{n}\right)^{*}$ with $H_{f}(A)=\left\{x_{0}\right\}$ and $H_{f}(B)=\left\{y_{0}\right\}$, then the pair $(A, B)$ is not minimal.

Proof. Assume that $f\left(x_{0}\right)=f\left(y_{0}\right)=0$. Let $\varepsilon>0$ and choose neighborhoods $U$ and $V$ of $x_{0}, y_{0}$ such that for all $x \in U \cap \partial A$ we have $x \in$ $z(x)+\varepsilon \mathbb{B}^{n} \subset A$ and for all $y \in V \cap \partial B$ we have $x \in w(y)+\varepsilon \mathbb{B}^{n} \subset B$, for some $z(x), w(y) \in \mathbb{R}^{n}$. There exists $\delta>0$ such that $\partial A \cap f^{-1}([-\delta, 0]) \subset U$ and $\partial B \cap f^{-1}([-\delta, 0]) \subset V$. Now, let $H^{-}=f^{-1}((-\infty,-\delta]), C=\operatorname{cl} \operatorname{conv}\{z(x) \mid$ $x \in \partial A \cap U\}$ and $D=\operatorname{cl} \operatorname{conv}\{w(y) \mid y \in \partial B \cap V\}$. Then

$$
A=\left(A \cap H^{-}\right) \cup\left(C+\varepsilon \mathbb{B}^{n}\right) \text { and } B=\left(B \cap H^{-}\right) \cup\left(D+\varepsilon \mathbb{B}^{n}\right),
$$

and it follows from Theorem 3.3 that the pair $(A, B)$ is not minimal.

Theorem 3.5 implies that the pair $(A, C)$ shown in Figure 1.2 is not minimal. In the following example we consider pairs of rotated lenses.

Example 3.6. For $x \in \mathbb{R}^{n}, n \geq 2$, with $\|x\|=1 / \sqrt{2}$ we define the $(n-2)$-dimensional sphere

$$
\begin{aligned}
S(x) & =\left\{y \in \mathbb{R}^{n} \mid\|y-x\|=\|y+x\|=1\right\} \\
& =\left\{y \in \mathbb{R}^{n} \mid\langle x, y\rangle=0 \text { and }\|y\|=1 / \sqrt{2}\right\} .
\end{aligned}
$$

The sphere $S(x)$ has center 0 and radius $1 / \sqrt{2}$ and is contained in the hyperplane perpendicular to $x$. Define a cigar to be

$$
C(x)=\bigcap\left\{y+\mathbb{B}^{n} \mid y \in S(x)\right\}
$$

and a flying saucer to be

$$
F(x)=\left(x+\mathbb{B}^{n}\right) \cap\left(-x+\mathbb{B}^{n}\right) .
$$

Notice that $C(-x)=C(x)$ and $F(-x)=F(x)$. The perpendicular projection of the pair $(F(x), C(x))$ onto any two-dimensional subspace of $\mathbb{R}^{n}$ containing $x$ is a pair of lenses illustrated in Figure 1.1. Since any such projection is a minimal pair, the pair $(F(x), C(x))$ itself is minimal.
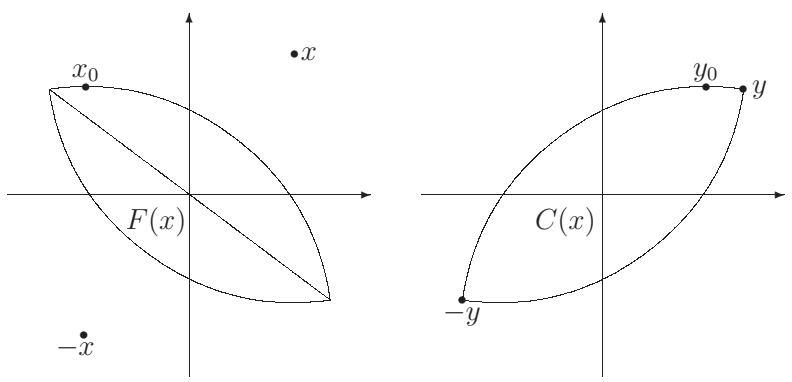

Fig. 3.4 
Now we prove that for $y \neq \pm x$ the pair $(F(x), C(y))$ is not minimal. Since $\|x\|=\|y\|=1 / \sqrt{2}$ the vectors $(x+y) /\|x+y\|$ and $(x-y) /\|x-y\|$ are orthogonal and of unit length. Hence the vector

$$
z=\frac{1}{\sqrt{2}}\left(\frac{x+y}{\|x+y\|}+\frac{x-y}{\|x-y\|}\right)
$$

is also of unit length. Put $f=\langle\cdot, z\rangle$. Let $\beta=\angle(x, x+y)$. Notice that $\beta \in(0, \pi / 2)$. Then $\angle(x, x-y)=\pi / 2-\beta$. Hence $\angle(x, z)<\pi / 4$. Therefore, $x_{0}=H_{f}(F(x))=H_{f}\left(-x+\mathbb{B}^{n}\right)=-x+z \notin S(x)$. Since for each $u \in$ $\partial F(x) \backslash S(x)$ and $\varepsilon>0$ the set $F(x)$ is locally $\varepsilon$-smooth at $u$, it follows that $F(x)$ is $\varepsilon$-smooth at $x_{0}$.

Set

$$
w=\frac{2\langle x, y\rangle y-x}{\sqrt{2}\|2\langle x, y\rangle y-x\|} .
$$

Notice that $w \perp y$ and $\|w\|=1 / \sqrt{2}$. We have $\beta=\angle(y, x+y)$ and $\angle(y, x-y)=\pi / 2+\beta$. Then $\angle(y, z)=\beta+\pi / 4 \in(\pi / 4,3 \pi / 4)$. Therefore, $y_{0}=H_{f}(C(y))=H_{f}\left(w+\mathbb{B}^{n}\right)=w+z \neq \pm y$. Since for each $u \in$ $\partial C(y) \backslash\{y,-y\}$ and $\varepsilon>0$ the set $C(y)$ is locally $\varepsilon$-smooth at $u$, we see that $C(y)$ is $\varepsilon$-smooth at $y_{0}$. It now follows from Theorem 3.5 that the pair $(F(x), C(y))$ is not minimal.

4. Reduction by subtraction. Let $X$ be a topological vector space and $A, B, C \in \mathcal{B}(X)$. Then $(A \dot{+} C, B \dot{+} C) \sim(A, B)$, which means that a pair can be reduced by subtracting common summands. If $X$ is locally convex there exists another reduction technique by cutting off by a hyperplane the common parts of $A$ and $B$ which can be translated onto each other by a vector (see [13, Theorem 4.7.3]). In this section we unify both reduction techniques.

Theorem 4.1. Let $A, A_{1}, A_{2}, B, B_{1}, B_{2}, C, D, F, G \in \mathcal{B}(X)$ with:

(i) $A=A_{1} \cup A_{2}, B=B_{1} \cup B_{2}$, where $A_{i}, B_{i} \in \mathcal{B}(X)$,

(ii) $A_{1}=C \dot{+} F, B_{1}=D \dot{+} F$,

(iii) $A_{1} \cap A_{2} \subset C \dot{+} G, B_{1} \cap B_{2} \subset D \dot{+} G$, where $G \subset F$.

Then $(A, B) \sim\left(A_{2} \cup(C \dot{+} G), B_{2} \cup(D \dot{+} G)\right)$.

Remark 4.2. The decomposition of the sets $A$ and $B$ in Theorem 4.1 is illustrated in Figure 4.1.

Proof. Notice that $A_{1} \cap A_{2} \subset(C \dot{+} G) \cap A_{2} \subset(C \dot{+} F) \cap A_{2}=A_{1} \cap A_{2}$. Since $C \dot{+} G \subset A_{1}$, the set $A_{1} \cap A_{2}$ separates $C \dot{+} G$ and $A_{2}$, and since $A_{1} \cap A_{2}=(C \dot{+} G) \cap A_{2}$, this implies that $C \dot{+} G$ and $A_{2}$ are separated by $(C \dot{+} G) \cap A_{2}$. Hence Theorem 2.2(ii) implies that $(C \dot{+} G) \cup A_{2}$ is convex. 


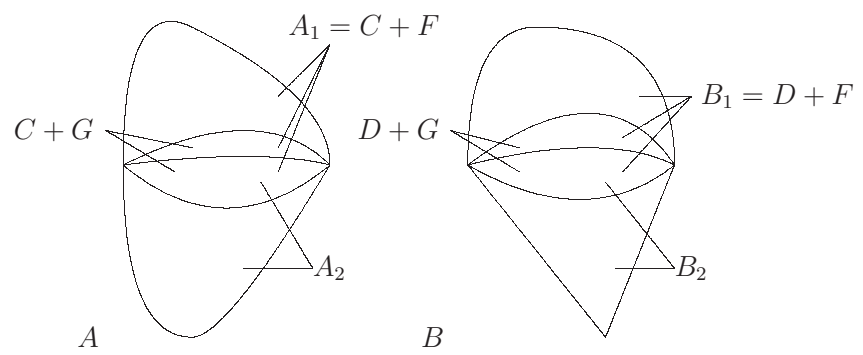

Fig. 4.1

Applying now Theorem 2.2(iii) to $A_{1} \cup A_{2}$ and $(C \dot{+} G) \cup A_{2}$ we obtain

$$
\begin{aligned}
F \dot{+}(C \dot{+} G) \cup A_{2} \dot{+} A_{1} \cap A_{2} & =F \dot{+} C \dot{+} G \dot{+} A_{2} \\
& =G \dot{+} A_{1} \dot{+} A_{2}=G \dot{+} A_{1} \cup A_{2} \dot{+} A_{1} \cap A_{2} \\
& =G \dot{+} A \dot{+} A_{1} \cap A_{2} .
\end{aligned}
$$

Now it follows from the cancellation law that $F \dot{+}(C \dot{+} G) \cup A_{2}=G \dot{+} A$. By a similar argument, $F \dot{+}(D \dot{+} G) \cup B_{2}=G \dot{+} B$. Therefore,

$$
\left((C \dot{+} G) \cup A_{2}, A\right) \sim(G, F) \sim\left((D \dot{+} G) \cup B_{2}, B\right),
$$

and consequently $(A, B) \sim\left(A_{2} \cup(C \dot{+} G), B_{2} \cup(D \dot{+} G)\right)$.

Theorem 4.3. Let $X$ be a locally convex vector space, $A, B, C, D, F \in$ $\mathcal{B}(X)$ and $f \in X^{*} \backslash\{0\}$ with $0 \in f(A) \cap f(B)$. Put $H^{+}=f^{-1}([0, \infty))$. If

$$
F \dot{+} C=A \cap H^{+} \text {and } F \dot{+} D=B \cap H^{+} \text {, }
$$

then $(A, B) \sim\left(A \cap H^{-} \cup\left(C \dot{+} H_{-f}(F)\right), B \cap H^{-} \cup\left(D \dot{+} H_{-f}(F)\right)\right.$.

Proof. Put $A_{1}=A \cap H^{-}, A_{2}=A \cap H^{+}, B_{1}=B \cap H^{-}, B_{2}=B \cap H^{+}$ and $G=H_{-f}(F)$. Then

$$
A_{1} \cap A_{2}=H_{-f}\left(A_{2}\right)=H_{-f}(F \dot{+} C)=H_{-f}(F) \dot{+} H_{-f}(C) \subset G \dot{+} C
$$

and hence $A_{1} \cap A_{2} \subset G \dot{+} C$. In a similar way we deduce that $B_{1} \cap B_{2} \subset G \dot{+} D$. Now the assertion follows from Theorem 4.1.

REMARK 4.4. Theorem 4.3 provides a combined reduction technique by subtracting the same summand and removing the common part of both sets which can be cut off by a hyperplane. It is the second main result of this paper and unifies and generalizes the two main known reduction techniques for pairs of bounded closed convex sets (see [13]).

ExAmple 4.5 . Let $T$ be a triangle in $\mathbb{R}^{2} \times\{0\}$ with center $(0,0,0)$, $S=-T, a=(0,0,1), b=(0,0,-1) \in \mathbb{R}^{3}$, and $H^{+}=\mathbb{R}^{2} \times[0, \infty)$. Define $A=b \vee 3 T \vee(2 a+T)$ and $B=b \vee(2 T+S) \vee(2 a+S)$.

Note that $A \cap H^{+}=F+T$ and $B \cap H^{+}=F+S$, where $F=2 T \vee 2 a$. Then the pair $(A, B)$ is equivalent to $\left(A \cap H^{-}, B \cap H^{-}\right)$. 

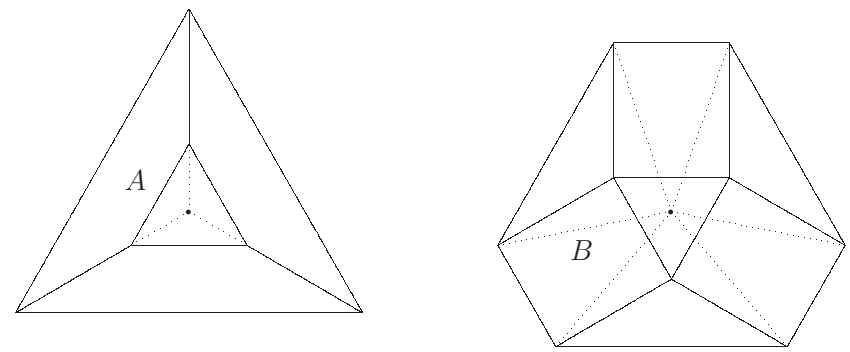

Fig. 4.2

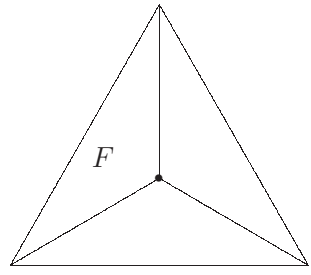

Fig. 4.3
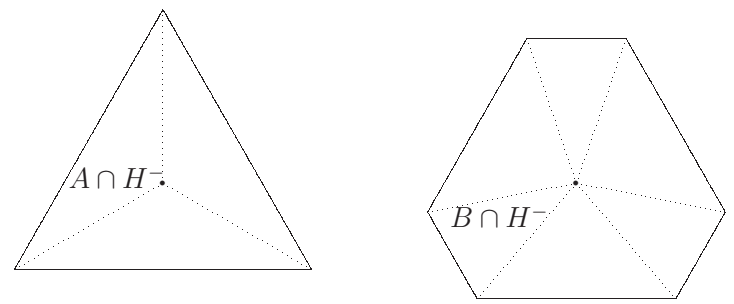

Fig. 4.4

\section{References}

[1] V. F. Demyanov and A. M. Rubinov, Quasidifferential Calculus, Optimization Software, New York, 1986.

[2] —, -, Quasidifferentiability and Related Topics, Nonconvex Optim. Appl., Kluwer, Dordrecht, 2001.

[3] V. F. Demyanov and E. Caprari, Minimality of convex sets equivalent on a cone, Vestnik St. Petersburg Univ. Mat. 31 (1998), 28-33 (in Russian).

[4] J. Grzybowski, Minimal pairs of compact convex sets, Arch. Math. (Basel) 63 (1994), 173-181.

[5] J. Grzybowski and R. Urbański, Minimal pairs of bounded closed convex sets, Studia Math. 126 (1997), 95-99.

[6] -, - On the number of minimal pairs of compact convex sets that are not translates of one another, ibid. 158 (2003), 59-63.

[7] L. Hörmander, Sur la fonction d'appui des ensembles convexes dans un espace localement convexe, Ark. Mat. 3 (1954), 181-186.

[8] D. Pallaschke, H. Przybycień and R. Urbański, On partially ordered semigroups and an abstract set-difference, Set-Valued Anal. 16 (2007), 257-265.

[9] D. Pallaschke and S. Rolewicz, Foundations of Mathematical Optimization-Convexity without Linearity, Math. Appl. 388, Kluwer, Dordrecht, 1997.

[10] D. Pallaschke, W. Urbańska and R. Urbański, C-minimal pairs of compact convex sets, ibid. 4 (1997), 1-25.

[11] D. Pallaschke and R. Urbański, A continuum of minimal pairs of compact convex sets which are not connected by translations, J. Convex Anal. 3 (1996), 83-95.

[12] - - - On the separation and order law of cancellation for bounded sets, Optimization 51 (2002), 487-496. 
[13] D. Pallaschke and R. Urbański, Pairs of Compact Convex Sets-Fractional Arithmetic with Convex Sets, Math. Appl. 548, Kluwer, Dordrecht, 2002.

[14] A. G. Pinsker, The space of convex sets of a locally convex space, Trudy Leningrad. Inzhen.-Ekonom. Inst. 63 (1966), 13-17 (in Russian).

[15] H. Rådström, An embedding theorem for spaces of convex sets, Proc. Amer. Math. Soc. 3 (1952), 165-169.

[16] H. Ratschek and G. Schröder, Representation of semigroups as systems of compact convex sets, ibid. 65 (1977), 24-28.

[17] S. Scholtes, Minimal pairs of convex bodies in two dimensions, Mathematika 39 (1992), 267-273.

[18] R. Urbański, A generalization of the Minkowski-Rådström-Hörmander theorem, Bull. Acad. Polon. Sci. Sér. Sci. Math. Astronom. Phys. 24 (1976), 709-715.

[19] -, On minimal convex pairs of convex compact sets, Arch. Math. (Basel) 67 (1996), 226-238.

[20] W. Weil, Eine Charakterisierung von Summanden konvexer Körper, Arch. Math. (Basel) 34 (1980), 283-288.

Faculty of Mathematics

and Computer Science

Adam Mickiewicz University

Umultowska 87

PL-61-614 Poznań, Poland

E-mail: jgrz@amu.edu.pl rich@amu.edu.pl
Institut für Statistik und Mathematische Wirtschaftstheorie Universität Karlsruhe Kaiserstr. 12 D-76128 Karlsruhe, Germany E-mail: lh09@rz.uni-karlsruhe.de

Received November 28, 2005

Revised version July 15, 2008 